BULL. AUSTRAL. MATH. SOC.

VOL. $23(1981), 367-381$.

\title{
TOLERANCE RELATIONS ON LATTICES
}

\author{
Hans-J, Bandelt
}

\begin{abstract}
The lattice of all tolerance relations (that is, reflexive, symmetric compatible relations) on a lattice is investigated. For modular lattices some examples are given which show that such relations do naturally occur.
\end{abstract}

A tolerance relation on a lattice is a reflexive and symmetric binary relation satisfying the Substitution Property (with respect to the lattice operations). Tolerance relations have been studied by Chajda, Zelinka, and others during the past decade. Although there are now numerous articles about tolerance relations on lattices, it has hardly ever been made clear how tolerance relations are related to the structure theory of lattices, nor why they deserve any interest. Now, tolerance relations "generalize" congruence relations (drop transitivity), but this would hardly serve to motivate them. For, there is no analogue amongst tolerance relations to the relationship of congruences and homomorphisms. As we see it there are two places where tolerance relations do arise naturally: the first is the study of (local) polynomial functions on lattices, and the second is the study of convex sublattices maximal with respect to certain properties. Let us commence with the first aspect. A polynomial function is any $n$-ary operation which is built up by the lattice operations and constants. Kindermann proved the following result: an order-preserving $n$-ary operation on a finite lattice is a polynomial function if and only if it preserves all tolerance relations; in particular, every orderpreserving function is a polynomial function if and only if the finite

Received 12 December 1980. 
lattice is "tolerance simple", that is, there are at most two (trivial) tolerance relations. It is quite clear that $1 t$ is much easier to determine whether a lattice has a proper tolerance relation than to test all order-preserving functions. It is the second aspect which serves as the main motivation for our present work. For a given lattice, we are interested in convex sublattices endowed with certain properties, either in their own right or since they form (in some sense) the building blocks of the lattice. If the given property is hereditary to subintervals, one may consider maximal convex sublattices with the property. It rarely happens that they actually form the blocks (alias classes) of some congruence relation; in general, they overlap, like maximal relatively complemented convex sublattices of a modular lattice or maximal $\alpha$-complete convex sublattices of a distributive lattice. Nevertheless, these "blocks" share some properties with congruence blocks, for instance, intervals which are projective into some block must also belong to some block. In either case, this is governed by a tolerance relation, where two elements are related if and only if they belong to a common block.

The organisation of the material is as follows. The first section provides the basic tools to decide whether a given binary relation on a lattice is a tolerance relation. In Section 2 the lattice of all tolerance relations is investigated. In particular, this lattice turns out to be pseudocomplemented, and (at least in the modular case) there is a convenient description of pseudocomplements. This is applied in the final section to some "canonical" tolerance relations arising in modular and distributive lattices. Some results of Herrmann, Kurinnot, and Jakubik can be seen in this light.

\section{Basic facts}

A reflexive and symmetric binary relation $\xi$ on a lattice $L$ is called a tolerance relation if $\xi$ is compatible, that is $\xi$ is a sublattice of $L^{2}$. $\Xi(L)$ denotes the lattice of all tolerance relations on - $L$ and $\theta(L)$ denotes the congruence lattice of $L$.

In order to prove that a given binary relation is a tolerance relation, Lemma 1.1 often facilitates the computations involved. This is, of course, analogous to the corresponding fact for congruence relations 
(see [9], Lemma I.3.8).

LEMMA 1.1. A reflexive binary relation $\xi$ on $L$ is a tolerance relation if and only if the following five properties are satisfied for $t, x, y \in L:$

(1) $(x, y) \in \xi$ if and only if $(x \wedge y, x \vee y) \in \xi$,

(2) $(t, x),(t, y) \in \xi$, and $t \leq x, y$ imply that $(t, x \vee y) \in \xi$,

(3) $(t, x),(t, y) \in \xi$, and $x, y \leq t$ imply that $(t, x \wedge y) \in \xi$,

(4) $(t, x) \in \xi$ and $x \leq t, y$ imply that $(t \vee y, y) \in \xi$,

(5) $(t, y) \in \xi$ and $t, x \leq y$ imply that $(t \wedge x, x) \in \xi$.

Proof. It is quite easy to see that every tolerance relation $\xi$ has properties (1) through (5). Assume now that $\xi$ is a reflexive binary relation satisfying $(1)-(5)$. Symmetry is trivial. Suppose $x, y$ belong to an interval $[u, v]$ where $(u, v) \in \xi$. Then by $(4),(x \wedge y, v) \in \xi$,

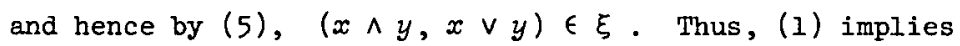

(6) $(u, v) \in \xi$ and $x, y \in[u, v]$ imply that $(x, y) \in \xi$.

Let $(u, v),(x, y) \in \xi$; we claim that $(u \vee x, v \vee y) \in \xi$. Indeed, we have $(u \wedge v, u \vee v),(x \wedge y, x \vee y) \in \xi$ by (1), and thus by (4),

$$
\begin{gathered}
((u \wedge v) \vee(x \wedge y), u \vee v \vee(x \wedge y)), \\
((u \wedge v) \vee(x \wedge y),(u \wedge v) \vee x \vee y) \in \xi .
\end{gathered}
$$

Applying (2), we get

$$
((u \wedge v) \vee(x \wedge y), u \vee v \vee x \vee y) \in \xi .
$$

Since $u \vee x, v \vee y \in[(u \wedge v) \vee(x \wedge y), u \vee v \vee x \vee y]$, we conclude using (6) that $(u \vee x, v \vee y) \in \xi$. Similarly, using (3), (5), (6), one proves that $(u \wedge x, v \wedge y) \in \xi$.

The lemma is best explained by Figure 1, that is, a reflexive (and symetric) relation $\xi$ is seen to be a tolerance relation if and only if it passes the following test: whenever there are pairs in $\xi$ labelled by " $\sim$ " in the six Hasse diagrams, then in either case the pair $(a, b)$ must belong to $\xi$. Note that it. is necessary to make the checks corresponding to each of the diagrams. 

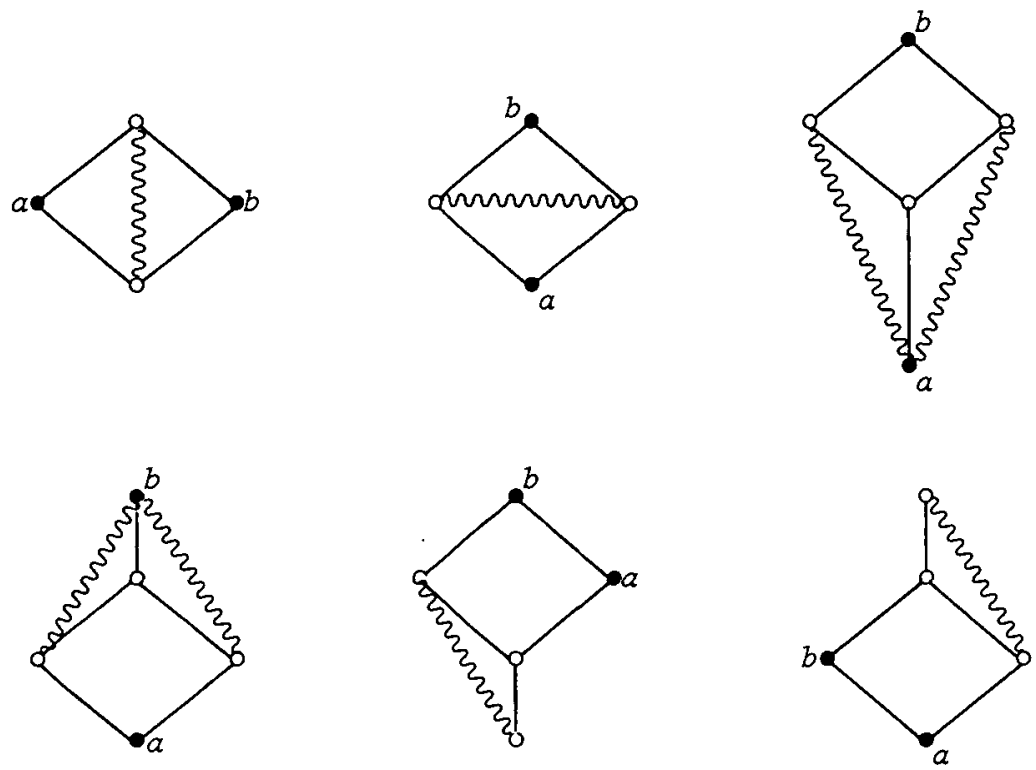

FIGURE 1

For a subset $A$ of $L$ and a tolerance relation $\xi$, let $A \xi$ denote the set of all $b \in L$ such that $(a, b) \in \xi$ for some $a \in A$. Whenever $A$ is a convex sublattice, so is $A \xi$. For, if $a, b \in A$ and $(a, x),(b, y) \in \xi$, then for $t \in[x \wedge y, x \vee y]$, we have $s=(t \vee(a \wedge b)) \wedge(a \vee b) \in A$ and $(s, t) \in \xi$.

Every subset $A$ of $L$ which is maximal with respect to $A^{2} \subseteq \xi$ is called a block of $\xi$. Obviously, $A$ is a block of $\xi$ if and only if $A$ is the intersection of all $\{a\} \xi$ where $a \in A$. Hence every block of $\xi$ is a convex sublattice of $L$ (cf. [1], Theorem 2). On the other hand, for a standard sublattice $S$ of $L$ (in the sense of [8]), the relation

$$
\begin{aligned}
& \theta[S]=\left\{(x, y) \in L^{2} \mid x \wedge y=((x \wedge y) \vee t) \wedge(x \vee y)\right. \text { and } \\
&x \vee y=((x \vee y) \wedge s) \vee(x \wedge y) \text { for some } s, t \in S\}
\end{aligned}
$$

is a congruence relation and $S$ is a block of $\theta[S]$ (see [8]). We assert that $\theta[S]$ is the least tolerance relation $\xi$ on $L$ such that $S^{2} \subseteq \xi$. Indeed, if $x \leq y$ in $L$ and $s, t \in S$ such that $x=(x \vee t) \wedge y$ and $y=(y \wedge s) \vee x$, then $(s, t) \in \xi$ and $x=(y \wedge t) \vee x$, whence 
$(x, y) \in \xi$. Note that this observation applies to [3], Theorem 3 , and to most results in [6].

To every tolerance relation $\xi$ on $L$ there corresponds the set $Q$ of all intervals $[a, b]$ of $L$ which are contained in some block of $\xi$, that is $(a, b) \in \xi$. The next lemma characterizes the sets $Q$ of intervals which give rise to tolerance relations. Recall that an interval $[x, y]$ is called weakly perspective into $[u, v]$ if either $u \leq x$ and $v \vee x=y$ or $y \leq v$ and $u \wedge y=x$ (cf. [9]).

LEMMA 1.2. Let $Q$ be a set of intervals such that $L=U Q$. The relation

$$
\xi_{Q}=\left\{(x, y) \in L^{2} \mid[x \wedge y, x \vee y] \in Q\right\}
$$

is a tolerance relation if and only if the following conditions are satisfied:

(7) if $[x, y]$ is weakly perspective into some $[u, v] \in Q$, then $[x, y] \in Q$,

(8) if $[t, x],[t, y] \in Q$, then $[t, x \vee y] \in Q$,

(9) if $[x, z],[y, z] \in Q$, then $[x \wedge y, z] \in Q$.

Proof. If $\xi_{Q}$ is a tolerance relation, then $(7),(8),(9)$ are trivially satisfied. Assume now that the set $Q$ satisfies (7), (8), (9). The relation $\xi_{Q}$ is symmetric by definition and is reflexive since $Q$ covers $L$ and (7) holds. Now, (1) is trivially true, (7) implies (2) and (3), (8) implies (4), (9) implies (5). Hence Lemma 1.1 completes the proof.

The relation $\xi_{Q}$ defined in Lemma 1.2 is a congruence relation if and only if the conditions

(10) $[x, y],[y, z] \in Q$ imply that $[x, z] \in Q$ and (7) are satisfied.

Lemmas 1.1 and 1.2 provide us with the necessary information required in Section 3 below. There are, however, many more ways to describe tolerance relations. For instance, every compatible relation containing the partial order $\leq$ corresponds to a tolerance relation, and vice versa 
(see [1]). Proposition 1.3 offers a characterization of tolerance relations in terms of certain mappings on.the ideal lattice $J(L)$. (Principal ideals are written as $(a](a \in L)$.)

PROPOSITION 1.3. For a lattice $L$, there is a 1-1 correspondence between tolerance relations $\xi$ and those mappings $\tau: J(L) \rightarrow J(L)$ which satisfy $A \subseteq A \tau$ for all ideals $A$ of $L$ and preserve finite intersections and (nonvoid) directed unions.

Proof. Given $\xi$, the mapping $\tau: A \mapsto A \xi \quad(A \in J(L))$ has the desired properties. Moreover, $\tau$ satisfies

(11) $(a, b) \in \xi$ if and only if $(a] \subseteq(b] \tau$ and $(b] \subseteq(a] \tau$. Conversely, given the mapping $\tau$, define a relation $\xi$ by (11). It is readily verified that $\xi$ is a tolerance relation. Further, if $b \in(a) \tau$, then $(a \wedge b] \subseteq(b] \tau$ and $(b] \subseteq(a) \tau \cap(b) \tau=(a \wedge b) \tau$, whence $(a \wedge b, b) \in \xi$ and thus

$$
(a] \tau \subseteq(a] \xi=\underset{x \leq a}{\bigcup}\{x\} \xi \subseteq \underset{x \leq \alpha}{U}(x] \tau=(a] \tau
$$

Consequently, for every ideal $A$, we have

$$
A \tau=\bigcup_{a \in A}(a] \tau=\bigcup_{a \in A}(a] \xi=A \xi,
$$

completing the proof.

\section{The lattice $\Xi(L)$}

Very little is known about the tolerance lattice $\Xi(L)$ of a lattice $L$. Since $\Xi(L)$ can be regarded as a subalgebra lattice of a universal algebra, $\Xi(L)$ is algebraic (cf. Section 3 in [5]). What about identities? If $L$ is distributive, so is $\Xi(L)$ (see [5], Theorem 16). However, if $L$ is modular, then $\Xi(L)$ is in general not modular: consider the tolerance relations $\xi_{0}, \xi_{1}, \xi_{2}$ on the lattice $L$ given by Figure 2. The diagrams exhibit the blocks of $\xi_{0}, \xi_{1}, \xi_{2}$, and $\xi_{0} \cap \xi_{1}=\xi_{0} \cap \xi_{2}$. Since $\xi_{0} \vee \xi_{1}=\xi_{0} \vee \xi_{2}=L^{2}$, we have an $N_{5}$ sitting inside $\Xi(L)$. 

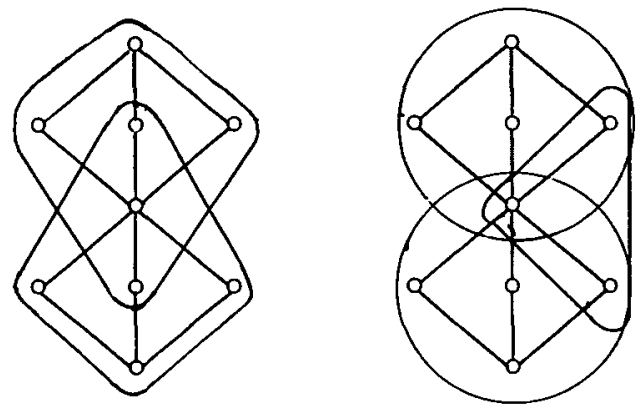

$\xi_{0}$

$\xi_{1}$
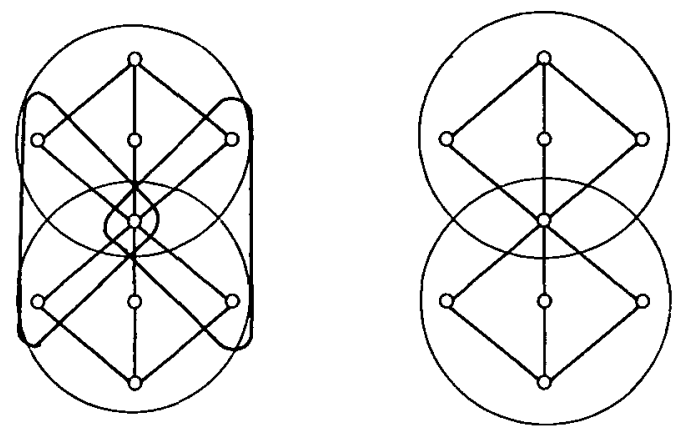

$\xi_{2}$

$\xi_{0} \cap \xi_{1}=\xi_{0} \cap \xi_{2}$

FIGURE 2

Therefore the variety of distributive lattices is the only nontrivial lattice variety $V$ for which all members have modular tolerance lattices. It might be true that the tolerance lattices of members in any nontrivial variety $V$ other than the variety of distributive lattices generate the variety of all lattices. At least, this "tolerance generated" variety must contain $V$. Indeed, every lattice $L$ is isomorphic to a sublattice of $\Xi(M)$, where $M$ is obtained by adjoining a new 0 to $L$; the desired embedding of $L$ in $\Xi(M)$ is given by $x \mapsto \xi_{x}=(x]^{2} \cup L^{2} \quad(x \in L)$.

We now proceed to prove the main result of this section. As we have already seen $\Xi(L)$ is in general far from being distributive. However, $\Xi(L)$ shares with $\theta(L)$ a local distributivity at the bottom $\omega$, that is $\Xi(L)$ is 0-modular and 0-distributive (in the sense of [15]). 
Recall that weak projectivity is the transitive extension of weak perspectivity: if for some natural number $n$ and

$$
[u, v]=\left[a_{0}, b_{0}\right],\left[a_{1}, b_{1}\right], \ldots,\left[a_{n}, b_{n}\right]=[x, y]
$$

every $\left[a_{i}, b_{i}\right]$ is weakly perspective into $\left[a_{i+1}, b_{i+1}\right]$ $(i=0, \ldots, n-1)$, then $[u, v]$ is weakly projective into $[x, y]$ (cf. [9]).

LEMMA 2.1. Given $\zeta \in \Xi(L)$ and $\theta \in \theta(L)$, let $Q$ be the set of all intervals $[x, y]$ such that $(u, v) \in \theta$ whenever $(u, v) \in \zeta$ and $[u, v]$ is weakly projective into $[x, y]$. Then $\xi_{Q}$ (defined as in Lemo 1.2) is a congruence relation and the relative pseudocomplement of $\zeta$ in $\theta$.

Proof. Clearly, $L$ is covered by $Q$ and (7) is true. To verify (10), let $[x, y],[y, z] \in Q$ and assume that $(u, v) \in \zeta$ and $[u$, v] is weakly projective into $[x, z]$. An easy induction shows that there exists $t \in[u, v]$ such that $[u, t]$ and $[t, v]$ are weakly projective into $[x, y]$ and $[y, z]$, respectively; for a single instance of weak perspectivity this is pictured in Figure 3 . Consequently, $(u, t)$ and $(t, v)$ belong to $\theta$, whence $(u, v)$ must be in $\theta$. We conclude that $\xi_{Q}$ is a congruence relation. By construction $\xi_{Q}$ is the largest tolerance relation with $\xi_{Q} \cap \zeta \subseteq \theta$, and we are done.

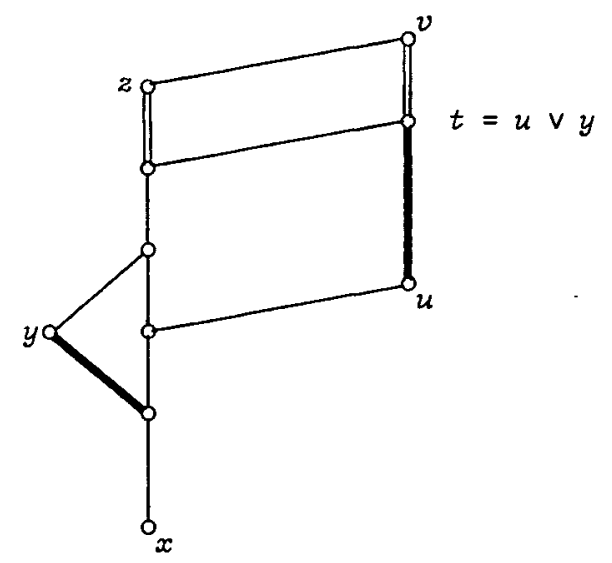

FIGURE 3 
In the presence of weak modularity, the pseudocomplement of a tolerance relation can be described in a more convenient way. A lattice $L$ is called weakly modular if for every proper interval $[r, \varepsilon]$ which is weakly projective into $[x, y]$, there exists a proper subinterval $[u, v]$ of $[x, y]$ which is weakly projective into $[r, s]$ (cf. [9]). As in the proof of [9, Theorem III.4.9] one obtains

LEMMA 2.2. Let $\xi$ be a tolerance relation on a weakly modular lattice $L$. The pseudocomplement $\xi^{*}$ of $\xi$ is a congmence relation and consists of all pairs $(x, y)$ such that $(u, v) \notin \xi$ for every proper subinterval $[u, v]$ of $[x \wedge y, x \vee y]$.

Proof. If $[x, y]$ is an interval which contains no pair $u<v$ with $(u, v) \in \xi$, then by weak modularity for every interval $[r, s]$ which is weakly projective into $[x, y]$, we must have $(r, s) \notin \xi$. Hence Lemma 2.1 finishes the proof.

LEMMA 2.3. Let $\xi_{0}$ and $\xi_{1}$ be tolerance relations on $L$ such that $\xi_{0} \cap \xi_{1}=\omega$ (that is, $\left.\xi_{1} \subseteq \xi_{0}^{*}\right)$. Then $\xi=\left\{(x, y) \in L^{2} \mid\right.$ for $i=0,1$, there exists $z_{i} \in L$ such that

$$
\left.x \wedge y=z_{0} \wedge z_{1}, x \vee y=z_{0} \vee z_{1},\left(x \wedge y, z_{i}\right) \in \xi_{i}\right\}
$$

is the join of $\xi_{0}$ and $\xi_{1}$ in $\equiv(L)$.

Proof. Since $\xi_{0}, \xi_{1} \subseteq \xi \subseteq \xi_{0} \vee \xi_{1}$, it suffices to verify that $\xi$ is a tolerance relation. We will check properties (1) through (5). (1) is trivial. Since $\xi_{0} \cap \xi_{1}=\omega,(x, y) \in \xi$ if and only if there is some selfaual diagram as given by Figure 4 .

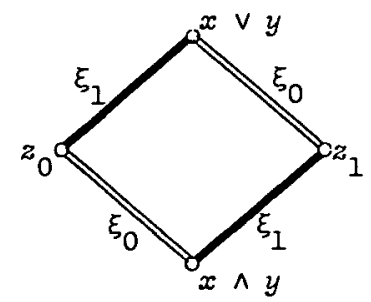

FIGURE 4 
Hence by duality we need only check (2) and (4). If $(t, x),(t, y) \in \xi$ and $t \leq x, y$, then there are $\left(t, u_{i}\right),\left(t, v_{i}\right) \in \xi_{i}(i=0,1)$ such that

$$
t=u_{0} \wedge u_{1}=v_{0} \wedge v_{1}, x=u_{0} \vee u_{1}, y=v_{0} \vee v_{1} \text {. }
$$

Then $\left(t, u_{i} \vee v_{i}\right) \in \xi_{i}(i=0,1)$ and $x \vee y=u_{0} \vee v_{0} \vee u_{1} \vee v_{1}$. Since $\left(t,\left(u_{0} \vee v_{0}\right) \wedge\left(u_{1} \vee v_{1}\right)\right) \in \xi_{0} \cap \xi_{1}=\omega$, we must have $t=\left(u_{0} \vee v_{0}\right) \wedge\left(u_{1} \vee v_{1}\right)$, whence $(t, x \vee y) \in \xi$, which proves (2). Now, if $(x, t) \in \xi$ and $x \leq t, y$, then there are $\left(x, z_{i}\right) \in \xi_{i}$ $(i=0,1)$ such that $x=z_{0} \wedge z_{1}$, and $t=z_{0} \vee z_{1}$. Then $\left(y, y \vee z_{i}\right) \in \xi_{i}(i=0,1)$ and $t \vee y=y \vee z_{0} \vee z_{1}$. Since $\left(y,\left(y \vee z_{0}\right) \wedge\left(y \vee z_{1}\right)\right) \in \xi_{0} \cap \xi_{1}=\omega$, we must have $y=\left(y \vee z_{0}\right) \wedge\left(y \vee z_{1}\right)$, whence $(y, t \vee y) \in \xi$, which proves (4). By Lemma 1.1, the proof is complete.

A lattice $L$ is called o-modular if it contains no $\mathrm{N}_{5}$ with bottom element 0 , that is, for $x, y, z \in L, x \leq y$ and $y \wedge z=0$ imply that $x=(x \vee z) \wedge y \quad($ ef. [15]).

THEOREM 2.4. For every lattice $L$, the tolerance lattice $\mathbf{\Xi}(L)$ is o-modular, pseudocomplemented and algebraic.

Proof. It remains to check 0-modularity. Let $\xi_{0}, \xi_{1}, \xi_{2} \in \Xi(L)$ such that $\xi_{1} \subseteq \xi_{2}, \xi_{0} \cap \xi_{2}=\omega$, and $\xi_{0} \vee \xi_{1}=\xi_{0} \vee \xi_{2}$. If $(x, z) \in \xi_{2}$ and $x<z$, then $(x, z) \in \xi_{0} \vee \xi_{1}$, whence by Lemma 2.3 there are $y_{0}, y_{1} \in L$ such that $x=y_{0} \wedge y_{1}, z=y_{0} \vee y_{1}$, and $\left(x, y_{i}\right) \in \xi_{i}(i=0,1)$. Since $\left(x, y_{0}\right) \in \xi_{0} \cap \xi_{2}=\omega$, we have $x=y_{0}$ and thus $y_{1}=z$. Hence $(x, z) \in \xi_{1}$, and therefore $\xi_{1}=\xi_{2}$. Consequently, $\Xi(L)$ is O-modular.

The lattice $\Delta(L)$ of all compatible reflexive relations is isomorphic to the square $(\Xi(L))^{2}$ (see [1]). Hence $\Delta(L)$ is also 0-modular and pseudocomplemented. 
Note that every finite distributive lattice is isomorphic to a tolerance lattice. This is a corollary to [9, Theorem II.3.17]: every finite distributive lattice is isomorphic to the congruence lattice of a sectionally complemented lattice. Indeed, on a sectionally complemented lattice there are no tolerance relations other than congruence relations (cf. [1]).

\section{Examples}

We will now demonstrate that there are several canonical tolerance relations on any modular lattice. On a distributive lattice one can find even more.

First some terminology. A lattice $L$ is atomistic if every element of $L$ is the join of atoms; $L$ is relatively atomistic if every interval of $L$ is an atomistic lattice. $L$ is discrete if every bounded chain is finite. $L$ is weakly atomic if every proper interval contains two elements $u$ and $v$ such that $v$ covers $u$; if $L$ does not contain such a pair, then $L$ is dense.

THEOREM 3.1. For a modular lattice $L$, define

$$
\begin{aligned}
& \theta=\left\{(x, y) \in L^{2} \mid[x \wedge y, x \vee y] \text { is of finite length }\right\}, \\
& \xi=\left\{(x, y) \in L^{2} \mid[x \wedge y, x \vee y] \text { is complemented }\right\} .
\end{aligned}
$$

Then $\theta$ is a congruence relation and $\xi$ is a tolerance relation on $L$. The blocks of

$$
\begin{aligned}
& \text { (i) } \theta, \\
& \text { (ii) } \theta^{*}, \\
& \text { (iii) } \theta^{* *}, \\
& \text { (iv) } \xi, \\
& \text { (v) } \theta^{* *} \cap \xi
\end{aligned}
$$

are the maximal convex sublattices of $L$ which are
(i) discrete,
(ii) dense,
(iii) weakly atomic, 
(iv) relatively complemented,

(v) relatively atomistic,

respectively.

Proof. It is not hard to see that $\theta$ is a congruence relation and that the blocks of $\theta, \theta^{*}, \theta^{* *}$ are of the asserted form. To prove that $\xi$, is a tolerance relation, let $a, b, c, x \in L$ such that $a \leq b, c$ and $a \leq x \leq b \vee c$. If $u$ is a relative complement of $b \wedge x$ in $[a, b]$ and if $v$ is a relative complement of $c \wedge(u \vee x)$ in $[a, c]$, then $u \vee v$ is a relative complement of $x$ in $[a, b \vee c]$. Indeed, by modularity, we have

$$
\begin{aligned}
(u \vee v) \wedge x & =(u \vee v) \wedge(u \vee x) \wedge x=[u \vee(v \wedge(u \vee x))] \wedge x \\
& =u \wedge x=a, \\
u \vee v \vee x & =[u \vee(b \wedge x)] \vee[v \vee(c \wedge(u \vee x))]=b \vee c .
\end{aligned}
$$

Hence, whenever $[a, b]$ and $[a, c]$ are complemented, so is $[a, b \vee c]$. Dually, $[a \wedge b, c]$ is complemented if $[a, c]$ and $[b, c]$ are complemented. By modularity, intervals which are weakly perspective into complemented intervals are complemented. Thus, applying Lemm 1.2, we get that $\xi$ is a tolerance relation. The blocks of $\xi$ are necessarily the maximal relatively complemented convex sublattices of $L$. It is routine to check that a complemented modular lattice is atomistic if and only if it is weakly atomic. Hence the blocks of $\theta^{* *} \cap \xi$ are the maximal relatively atomistic convex sublattices of $L$.

For a modular lattice $L$ of finite length, the blocks of the relation $\xi$ in Theorem 3.1 are the maximal atomistic intervals of $L$. These intervals were studied in [10], [13]. Some of the results in these papers follow (with remarks in [1]) from the fact that $\xi$ is a tolerance relation. If $L$ is a distributive lattice, then $\xi$ can be characterized as the intersection of all tolerance relations with exactly two overlapping blocks (cf. [2]).

Completeness conditions in distributive lattices also give rise to natural tolerance relations. A lattice $L$ is called < $\alpha$-complete if for every nonempty subset $A$ of $L$ with fewer than $\alpha$ elements, the join $\checkmark A$ and the meet $\wedge A$ exist. For a cardinal $\alpha, \alpha^{+}$denotes its successor cardinal. Then $L$ is a-complete if and only if $L$ is 
$<\alpha^{+}$-complete. $L$ is conditionalzy $<\alpha$-complete if every interval of $L$ is $<\alpha$-complete. $L$ is called < $\alpha$-homogeneous if $L$ is conditionally $<\alpha$-complete and does not contain any $\alpha$-complete proper interval.

PROPOSITION 3.2. Let $L$ be a distributive lattice and let $\alpha$ be an uncountable cardinal. Then

$$
\xi_{\alpha}=\left\{(x, y) \in L^{2} \mid[x \wedge y, x \vee y] \text { is < <-complete }\right\}
$$

is a tolerance relation. The blocks of $\xi_{\alpha}$ and $\xi_{\alpha} \cap\left(\xi_{\alpha}\right)^{*}$ are the maximal conditionally < $<$-complete convex sublattices and the maximal $<a-h o m o g e n e o u s$ convex sublattices of $L$, respectively.

Proof. Let $[x, y]$ and $[x, z]$ be $<\alpha$-complete intervals. Assume that $A$ is a nonempty subset of $[x, y \vee z]$ with fewer than $\alpha$ elements. Then

$$
u=\underset{a \in A}{\vee} y \wedge a \text { and } v=\vee_{a \in A}^{\vee} z \wedge a
$$

exist. Since $L$ is distributive, $u \vee v$ is an upper bound of $A$. If $w$ is any upper bound of $A$, then $u \leq y \wedge w$ and $v \leq z \wedge w$, whence $u \vee v \leq w$. We conclude that $u \vee v=\vee A$. Dually, if $[x, z]$ and $[y, z]$ are < $\alpha$-complete, then $[x \wedge y, z]$ is < $\alpha$-complete. It is readily seen that an interval which is weakly perspective into an $<\alpha$-complete interval is also < $\alpha$-complete. Thus an application of Lemmas 1.2 and 2.2 completes the proof.

Proposition 3.2 applies to the results in [11], $\$ 1,3$, which are proved for relatively complemented distributive lattices. In this case, of course, all the $\xi_{\alpha}$ are congruence relations. For arbitrary distributive lattices this does not hold (see [11], Example 1.8). For modular lattices, $\xi_{\alpha}$ may even fail to be a tolerance relation, as our final example shows. Consider the modular lattice $K=F M\left(M_{D}\right)$ of [14], where $D$ denotes the chain of integers with bounds added (see Figure 5, p. 380). By [14], Hilfssatz $2, D \cong[0, a] \cong[a, 1]$, and hence $a$ is doubly irreducible. Thus $L=K-\{a\}$ is a noncomplete modular lattice. Since $[0, b]$ and $[0, c]$ are complete and $b \vee c=1, \xi_{\aleph_{1}}$ is not a tolerance relation of $L$. 


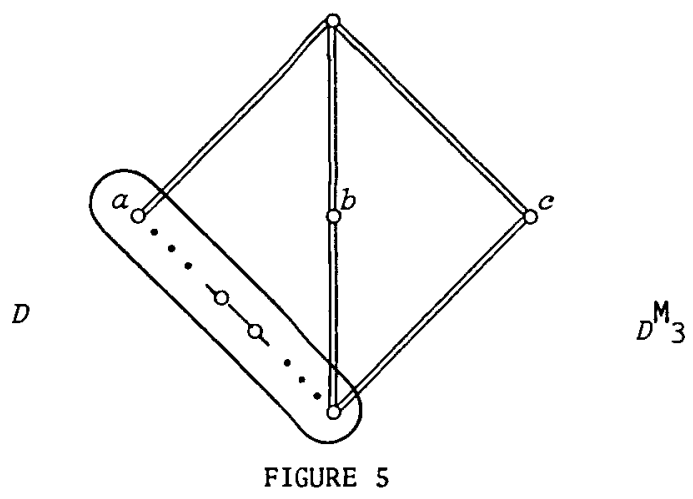

\section{References.}

[1] Hans-J. Bandelt, "Local polynomial functions on lattices", Houston $J$. Math. (to appear).

[2] Hans-J. Bandelt, "Tolerances on median algebras", submitted.

[3] I van Chajda, "Notes on lattice congruences", Časopis Pěst. Mat. 103 (1978), 255-258.

[4] Ivan Chajda, "A characterization of distributive lattices by tolerance lattices", Arch. Math. (Brno) 15 (1979), 203-204.

[5] Ivan Chajda and Bohdan Zelinka, "Lattices of tolerances", Casopis Pěst. Mat. 102 (1977), 10-24.

[6] I van Chajda and Bohdan Zelinka, "Minimal compatible tolerances on lattices", Czechoslovak Math. J. 27 (102) (1977), 452-459.

[7] I van Chajda and Bohdan Zelinka, "Tolerances and convexity", Czechoslovak Math. J. 29 (104) (1979), 584-587..

[8] E. Fried and E.T. Schmidt, "Standard sublattices", Algebra Universalis 5 (1975), 203-211.

[9] George Grätzer, General lattice theory (Birkhäuser Verlag, Basel and Stuttgart, 1978).

[10] Christian Herrmann, "S-verklebte Summen von Verbänden", Math. 2. 130 (1973), 255-274. 
[11] J. Jakubík, "Conditionally $\alpha$-complete sublattices of a distributive lattice", Algebra Universalis 2 (1972), 255-261.

[12] M. Kindermann, "Über die Äquivalenz von Ordnungspolynomvollständigkeit und Toleranzeinfachheit endlicher Verbände", Contributions to general algebra, 145-149 (Proc. Klagenfurt Conf., Klagenfurt, 1978. Verlag Johannes Heyn, Klagenfurt, 1979).

[13] Г.Ч. Нуринной [G.ð. Kurinno I], "Новое доназательство теоремы Днлуорса" [A new proof of a theorem of Dilworth], Vestnik Har'kov. Gos. Univ. No. 93 Mat. Vyp. 38 (1973), 11-15, 1.

[14] Aleit Mitschke und Rudolf Wille, "Freie modulare Verbände $F M\left(D M_{3}\right)$ ", Proceedings of the University of Houston Lattice Theory Conference, Houston, Texas, 1973, 383-396 (Department of Mathematics, University of Houston, Houston, Texas, 1973).

[15] J.C. Varlet, "A generalization of the notion of pseudocomplementedness", Bulz. Soc. Roy. Sci. Liège 37 (1968), 149-158.

Universität Ol denburg,

Fachbereich IV - Mathematik,

Ammerländer Heerstrasse 67-99,

Postfach 2503,

2900 Oldenburg,

F.R. Germany. 